\title{
VALIDATION OF CHEST COMPUTED TOMOGRAPHY ARTIFICIAL INTELLIGENCE TO DETERMINE THE REQUIREMENT FOR MECHANICAL VENTILATION AND RISK OF MORTALITY IN HOSPITALIZED CORONAVIRUS DISEASE-19 PATIENTS IN A TERTIARY CARE CENTER IN MEXICO CITY
}

\author{
Yukiyoshi Kimura-Sandoval ${ }^{1}$, Mary E. Arévalo-Molina², César N. Cristancho-Rojas ${ }^{2 *}$, \\ Yumi Kimura-Sandoval ${ }^{1}$, Victoria Rebollo-Hurtado ${ }^{2}$, Mariana Licano-ZUbiate ${ }^{1}$, \\ MónICA Chapa-IBARGÜENGOITIA ${ }^{1}$, AND GISELA MUÑOZ-LÓPEZ ${ }^{1}$
}

Department of Radiology, ${ }^{1}$ Instituto Nacional de Ciencias Médicas y Nutrición Salvador Zubirán, Mexico City and ${ }^{2} \mathrm{CT}$ Scanner Group, Mexico City, Mexico

\begin{abstract}
Background: Artificial intelligence (Al) in radiology has improved diagnostic performance and shortened reading times of coronavirus disease 2019 (COVID-19) patients' studies. Objectives: The objectives pf the study were to analyze the performance of a chest computed tomography (CT) Al quantitative algorithm for determining the risk of mortality/mechanical ventilation (MV) in hospitalized COVID-19 patients and explore a prognostic multivariate model in a tertiary-care center in Mexico City. Methods: Chest CT images of 166 COVID-19 patients hospitalized from April 1 to 20, 2020, were retrospectively analyzed using Al algorithm software. Data were collected from their medical records. We analyzed the diagnostic yield of the relevant CT variables using the area under the ROC curve (area under the curve [AUC]). Optimal thresholds were obtained using the Youden index. We proposed a predictive logistic model for each outcome based on CT Al measures and predetermined laboratory and clinical characteristics. Results: The highest diagnostic yield of the assessed CT variables for mortality was the percentage of total opacity (threshold $>51 \%$; AUC $=0.88$, sensitivity $=74 \%$, and specificity $=91 \%$ ). The AUC of the CT severity score (threshold $>12.5$ ) was 0.88 for MV (sensitivity $=65 \%$ and specificity $=92 \%$ ). The proposed prognostic models include the percentage of opacity and lactate dehydrogenase level for mortality and troponin I and CT severity score for MV requirement. Conclusion: The Al-calculated CT severity score and total opacity percentage showed good diagnostic accuracy for mortality and met MV criteria. The proposed prognostic models using biochemical variables and imaging data measured by $\mathrm{Al}$ on chest CT showed good risk classification in our population of hospitalized COVID-19 patients. (REV INVEST CLIN. 2021;73(2):111-9)
\end{abstract}

Key words: COVID-19. Artificial intelligence. Diagnostic imaging. Chest. Computed tomography.

*Corresponding author:

César N. Cristancho-Rojas

E-mail: cncr777@hotmail.com
Received for publication: 01-09-2020

Approved for publication: 13-10-2020

DOI: $10.24875 / R I C .20000451$

0034-8376 / (c) 2020 Revista de Investigación Clínica. Published by Permanyer. This is an open access article under the CC BY-NC-ND license (http://creativecommons.org/licenses/by-nc-nd/4.0/). 


\section{INTRODUCTION}

The coronavirus disease 2019 (COVID-19) pandemic continues to spread, confronting healthcare professionals worldwide ${ }^{1}$. Hospital overloading and a scarcity of mechanical ventilators and intensive care unit beds are critical concerns. The ultimate diagnosis of COVID-19 relies on reverse-transcriptase real-time polymerase chain reaction (RT-PCR $)^{2,3}$; however, the false-negative rate is high $(39-61 \%)^{4}$.

Thin-section chest computed tomography (CT) has a high sensitivity for the diagnosis of COVID-195,6. Our institution follows the Fleischner Society Consensus ${ }^{7}$ and the European Society of Radiology ${ }^{8}$ guidelines, which recommend using CT imaging in various clinical scenarios, including patients presenting moderate-tosevere symptoms consistent with those of COVID-19. Several visual assessments using semiquantitative $C T$ severity scores (CT-SS) with the degree of lung opacification have been proven significant in identifying patients with severe disease ${ }^{3}$.

The importance of radiological findings in COVID-19 patients has increased radiologists' workload, who must interpret more images promptly to avoid delays in diagnosis ${ }^{9}$ and facilitate resource allocation. Fortunately, the rapid development of artificial intelligence (Al) in the field of radiology has improved diagnostic performance and shortened reading times ${ }^{10}$, with very high sensitivity and specificity ${ }^{11}$. For instance, Lin et al. used 4356 chest CT scans to develop a three-dimensional learning model (COVNet) to differentiate correctly COVID-19 from community-acquired pneumonia with a sensitivity and specificity of $90 \%$ and $96 \%$, respectively ${ }^{12}$.

Some studies have described algorithms capable of identifying and quantifying abnormal tomographic patterns in non-contrast chest CT images of patients with COVID-199 with high sensitivity and specificity and excellent areas under the receiver operating characteristic (ROC) curve (area under the curve $[A U C]^{12}$. These automated systems perform segmentation of the lungs, lobes, and compromised lung parenchyma in 3D. Some measure the percentage of opacity and high opacity and calculate a CT severity score. This technology has opened the door for Al to classify risk, monitor patients' responses to treatment, and evaluate disease progression. However, few studies have compared the percentage and degree of lung opacification related to patient outcomes in selected populations.

This study aimed to analyze the classification performance of a chest CT Al quantitative algorithm in a cohort of hospitalized COVID-19 patients, establishing quick classification thresholds that could contribute to determine the mortality risk and the need for mechanical ventilation (MV). This study also constructed a prognostic multivariate logistic model testing $\mathrm{CT}$ Al measures and predetermined clinical and laboratory characteristics to validate this AI CT tool.

\section{METHODS}

The protocol of this observational retrospective study followed the Declaration of Helsinki's ethical requirements and was approved by the Institutional Review Board. Informed consent was waived because the institution's protocol had already acquired the images for these patients.

\section{Data sources}

Demographic, clinical, biochemical, and radiological data were obtained from electronic medical records of hospitalized COVID-19 patients from April 1-20, 2020, in a Tertiary Health Care Center of Mexico City.

The inclusion criteria were hospitalized adult ( $\geq 18$ years old) patients with a positive RT-PCR for SARSCoV 2 who had undergone non-contrast chest CT. Hospitalized patients with incomplete or unavailable $\mathrm{CT}$ images in the Picture Archiving and Communication System were excluded from the study. The patients' demographic data included age, sex, and comorbidities and laboratory data included leukocyte count, creatinine, lactate dehydrogenase, ferritin, C-reactive protein, high-sensitivity troponin I, and D-dimer levels.

The primary outcome variables were as follows: patient requirement for $\mathrm{MV}$ (defined as patients with no improvement in respiratory distress with a nonrebreathing mask with high flow [10-15 L/min], a respiratory rate higher than $30 / \mathrm{min}, \mathrm{PaO}_{2}<60 \mathrm{~mm} \mathrm{Hg}$, and $\mathrm{PaO}_{2} / \mathrm{FiO}_{2}$ [obtained from noninvasive respiratory 
Figure 1. Quantitative lung computed tomography (CT) analysis. (A) Non-contrast chest CT images in the axial, sagittal, and coronal planes show automatic lung segmentation and bilateral ground-glass detection by Al software. (B) Al software volumerendered images highlight compromised lung areas (mean attenuation > -700 Hounsfield units). (C) Overall total opacity score, total percentage of opacity, and other $\mathrm{CT}$ variables are shown in the results table.

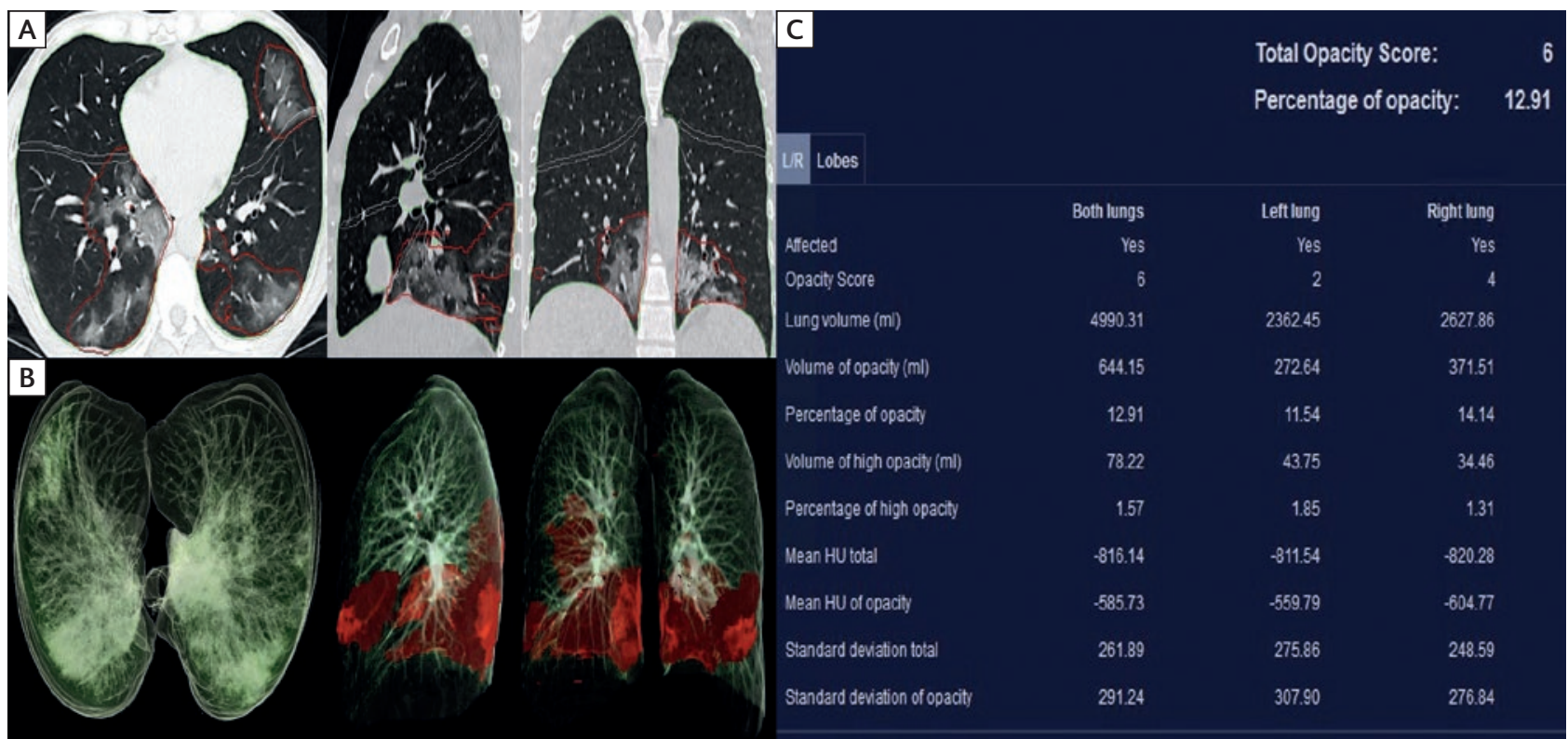

support $]<150$ with respiratory distress, or any patient with $\mathrm{PaO}_{2} / \mathrm{FiO}_{2}<100$ ), and all-cause mortality during hospitalization.

\section{Chest CT scan and quantitative analysis}

All chest CT scans were performed using a 64-detector CT scanner (Revolution EVO, General Electric) with the following parameters: $120 \mathrm{kV}, 350 \mathrm{mAs}$, rotation time $0.4 \mathrm{~s}$, pitch 1.5 , and intersection space 5 $\mathrm{mm}$. Furthermore, reconstructions using a $1.5-\mathrm{mm}$ slice thickness and sharp convolution kernel were performed. All CT scans were performed with the patient in a supine position. The field of view included the whole chest (from the lung apex to the suprarenal glands) and was obtained during forced inspiration. The entire dataset was anonymized and exported in Digital Imaging and Communication on Medicine format for segmentation and quantification. Using CT pneumonia analysis prototype software (Siemens Healthcare version 30, Erlangen, Germany), an Al algorithm based on three-dimensional segmentation automatically detected and quantified abnormal tomographic patterns (ground-glass opacities and consolidations) in each and both lung parenchyma based on deep learning and deep reinforcement learning. This Al algorithm was previously trained in controls and a large group of patients with diseases (average report time of 1-3 min per chest CT examination).

CT-SS, volume and percentage of opacity, and volume and percentage of high opacity were automatically processed by the Al software (Fig. 1). All post-processing results were reviewed, manually corrected, and approved by a general radiologist with more than 10-years' experience. CT-SS is calculated by dividing the lung parenchyma into five anatomical lobes and assigning scores (0-20) based on the percentage of opacity within the lobes $(0,1,2,3$, and 4 if parenchymal opacification was $0,<25 \%,<50 \%,<75 \%$, and $\geq 75 \%$, respectively). Volumes of opacity and high opacity were calculated based on Hounsfield units ( $\mathrm{HU}$; areas with mean densities higher than $-700 \mathrm{HU}$ and -200 , respectively) ${ }^{13}$, and percentages were calculated dividing the compromised volume by the total measured pulmonary volume.

\section{Statistical analysis}

Continuous variables were expressed as means and standard deviations or medians with interquartile 
ranges, and categorical variables were expressed as percentages and absolute frequencies. Subsequently, we performed bivariate logistic correlation (each independent variable vs. the outcomes reporting crude odds ratio $[\mathrm{ORc}]$ ) to establish the significance as a prerequisite for inclusion in the multivariate model.

We tested the diagnostic yield of the relevant variables obtained from CT images (CT-SS, the total volume of opacity and high opacity, and percentage of opacity and high opacity) using a ROC-based analysis, with their AUC and 95\% confidence intervals (Cl). Optimal thresholds were obtained using the Youden Index (J) to describe the sensitivity, specificity, positive predictive value, negative predictive value, positive likelihood ratio, and negative likelihood ratio. All hypothesis tests considered a significant two-tailed $p<0.05$.

We performed a multivariate analysis with logistic regression to identify the predictive model for each outcome (meeting criteria for MV and mortality) based on CT variables, including predetermined significant correlated clinical and laboratory variables (age, diabetes, hypertension, leukocyte count, and C-reactive protein, ferritin, D-dimer, high-sensitivity troponin I, and lactate dehydrogenase levels) that were previously reported in original research in an identical population. The final parameter reported was the adjusted risk ratio: ORa ${ }^{14}$. Hosmer-Lemeshow tests were performed to test for the goodness of fit of the logistic regression models (calibration), with overall (Brier score) and discrimination (C score) statistics. The analysis was performed on STATA SE version 14.1 software and SPSS software package version 20.

\section{RESULTS}

\section{Demographic and clinical variables}

The median age of the 166 patients who met the inclusion criteria was $50 \pm 14$ years and $60.2 \%$ were men. The outcomes, all-cause mortality and meeting criteria for MV, were observed in $21.08 \%$ and $30.72 \%$ of patients, respectively. Patients' demographic data are presented in table 1 .
Table 1. Patients' demographic data* $(n=166)$

\begin{tabular}{|c|c|}
\hline Variables & Result \\
\hline Age, years mean (SD) & $50(14)$ \\
\hline Male (\%) & $100(60.2)$ \\
\hline $\begin{array}{l}\text { Patient meeting criteria } \\
\text { for MV (\%) }\end{array}$ & $51(30.72)$ \\
\hline Patient with access to $\mathrm{MV}^{* *}(\%)$ & $49(29.52)$ \\
\hline Global mortality (\%) & $35(21.08)$ \\
\hline Length of stay, days mean (SD) & $10(8.6)$ \\
\hline \multicolumn{2}{|l|}{ Comorbidities (\%) } \\
\hline Diabetes & $44(26.51)$ \\
\hline Hypertension & $39(23.49)$ \\
\hline COPD & $1(0.60)$ \\
\hline Smoker & $5(3.01)$ \\
\hline Asthma & $2(1.20)$ \\
\hline Obesity & $28(16.87)$ \\
\hline Immunosuppression & $4(2.41)$ \\
\hline Cardiovascular disease & $2(1.20)$ \\
\hline Cancer & $2(1.20)$ \\
\hline Organ transplant & $6(3.61)$ \\
\hline \multicolumn{2}{|l|}{ Laboratory data } \\
\hline $\begin{array}{l}\text { Leukocytes cells } / \mathrm{mm}^{3} \\
\text { mean (SD) }\end{array}$ & $8.5(6.8)$ \\
\hline Creatinine, $\mathrm{mg} / \mathrm{dL}$ mean (SD) & $0.95(0.36)$ \\
\hline $\begin{array}{l}\text { Lactate dehydrogenase, } \mathrm{UI} / \mathrm{dL} \\
\text { mean (SD) }\end{array}$ & $375(142.3)$ \\
\hline Ferritin, mg/dL median (IQR) & $627(577.4)$ \\
\hline CRP, mg/dL median (IQR) & $12.86(5.49-19.28)$ \\
\hline Troponin I, ng/dL median (IQR) & $4.95(3.3-9.4)$ \\
\hline D-Dimer, ng/dL median (IQR) & $584(401-1018)$ \\
\hline
\end{tabular}

SD: standard deviation; MV: mechanical ventilation; COPD: chronic pulmonary obstructive pulmonary disease; $\mathrm{CRP}$ : $\mathrm{C}$ reactive protein; IQR: interquartile range.

*Data presented as $\mathrm{n}(\%)$ unless specified.

**Patients without access to MV due to limited resources were not included.

\section{Optimal thresholds of CT variables for the classification of mortality and $\mathrm{MV}$ requirement}

The highest diagnostic yield of CT variables for the outcomes of interest was the percentage of total opacity with $A \cup C_{\text {mortality }}$ (threshold $>51 \%$ ) $=0.88$ (95\% Cl, 0.81-0.94) and $\mathrm{AUC}_{\mathrm{MV}}$ (threshold > 25\%) $=0.88(95 \% \mathrm{Cl}, 0.83-0.93)$, with sensitivity, specificity, and negative predictive values of $74 \%, 91 \%$, and 
Table 2. Diagnostic performance of CT AI calculated variables for COVID-19 pneumonia

\begin{tabular}{|c|c|c|c|c|c|c|c|c|c|c|}
\hline Outcome & Variable & AUC $(95 \% \mathrm{Cl})$ & Threshold & $\begin{array}{l}\text { Youden } \\
\text { index }\end{array}$ & SEN & SPE & PPV & NPV & $\mathrm{LR+}$ & LR- \\
\hline \multirow{5}{*}{$\begin{array}{l}\text { Mechanical } \\
\text { ventilation } \\
\text { criteria }\end{array}$} & Severity score & $0.884(0.83-0.937)$ & $>12.5$ & 0.56 & 65 & 92 & 78 & 85 & 8.26 & 0.38 \\
\hline & $\begin{array}{l}\text { Total opacity } \\
\text { volume }\end{array}$ & $0.843(0.783-0.903)$ & > $793 \mathrm{ml}$ & 0.55 & 86 & 69 & 55 & 91 & 2.75 & 0.19 \\
\hline & $\%$ total opacity & $0.886(0.837-0.936)$ & $>25 \%$ & 0.593 & 86 & 73 & 58 & 92 & 3.2 & 0.18 \\
\hline & $\begin{array}{l}\text { High opacity } \\
\text { volume }\end{array}$ & $0.87(0.814-0.926)$ & $>190 \mathrm{ml}$ & 0.56 & 75 & 82 & 64 & 87 & 4.08 & 0.31 \\
\hline & \% high opacity & $0.866(0.809-0.923)$ & $>8 \%$ & 0.56 & 65 & 91 & 76 & 85 & 7.44 & 0.38 \\
\hline \multirow[t]{5}{*}{ Mortality } & Severity score & $0.876(0.805-0.947)$ & $>10.5$ & 0.64 & 83 & 82 & 54 & 94 & 4.52 & 0.2 \\
\hline & $\begin{array}{l}\text { Total opacity } \\
\text { volume }\end{array}$ & $0.847(0.773-0.922)$ & $>1158 \mathrm{ml}$ & 0.59 & 83 & 76 & 48 & 94 & 3.5 & 0.22 \\
\hline & \% total opacity & $0.881(0.816-0.947)$ & $>51 \%$ & 0.65 & 74 & 91 & 68 & 93 & 8.1 & 0.28 \\
\hline & $\begin{array}{l}\text { High opacity } \\
\text { volume }\end{array}$ & $0.848(0.77-0.92)$ & $>165 \mathrm{ml}$ & 0.56 & 86 & 71 & 44 & 94 & 2.95 & 0.2 \\
\hline & \% high opacity & $0.834(0.762-0.907)$ & $>9 \%$ & 0.53 & 63 & 91 & 64 & 90 & 6.86 & 0.4 \\
\hline
\end{tabular}

Al: artificial intelligence; AUC: semiquantitative (area under the ROC curve); SEN: sensitivity; SPE: specificity; PPV: positive predictive value; NPV: negative predictive value; LR: likelihood ratio.

$93 \%$, respectively, for mortality and $86 \%, 73 \%$, and $92 \%$, respectively, for meeting MV criteria. For CT-SS, $A \cup C_{\text {mortality }}$ (threshold $\left.>10.5\right)=0.87(95 \% \mathrm{Cl}$, $0.80-0.94)$, with sensitivity, specificity, and negative predictive values of $83 \%, 82 \%$, and $94 \%$, respectively, and $\mathrm{AUC}_{\mathrm{MV}}$ (threshold > 12.5) $=0.88$ $(95 \% \mathrm{Cl}, 0.83-0.93)$, with sensitivity, specificity, and negative predictive values of $65 \%, 92 \%$, and $85 \%$, respectively. Data for the remaining CT variables and outcomes of interest are detailed in table 2. Figs. 2 and 3 show the ROC curves for mortality and $\mathrm{MV}$ requirement.

\section{Predictive logistic models for mortality and $\mathrm{MV}$ requirement}

In the first approximation, we calculated ORc as part of the previous bivariate analysis for model construction and found statistical significance in the correlation of CT variables with mortality and MV requirement (Table S1). Conversely, there were clinical and laboratory variables that were not significantly correlated with mortality (diabetes [ $p=0.24$ ], hypertension [ $=0.09]$, leukocyte count $[p=0.18]$, ferritin $[p=0.09]$, and D-dimer $[p=0.096])$ or MV requirement (age $[p=0.23]$, diabetes $[p=0.34]$, hypertension $[p=0.43]$, leukocyte count $[p=0.21]$, ferritin $[p=0.11]$, and D-dimer $[p=0.099]$ ). Hence, we excluded these variables from the predictive model.

In the first multivariate logistic analysis (Enter Method), which included clinical characteristics (age, lactate dehydrogenase, $\mathrm{C}$-reactive protein, and troponin I) and the CT values, the adjusted OR (ORa) lost significance, except for CT-SS ( $p=0.007)$ and tropo$\operatorname{nin} I(p=0.017)$ for MV requirement, and the percentage of total opacity $(p=0.02)$ and lactate dehydrogenase $(p=0.034)$ for mortality. A second model for mortality showed a lack of goodness of fit $(p=0.05)$; thus, we transformed the percentage of total opacity into a dichotomic variable based on the threshold obtained using the Youden test $(>51 \%)$, achieving goodness of fit with a slightly better $R^{2}$ (0.368 vs. 0.386). Table $S 2$ details the proposed prognostic model and predicting equations for meeting the MV criteria, and table $\mathrm{S} 3$ presents the same for mortality.

\section{DISCUSSION}

Quantitative $\mathrm{Al}$ analysis of CT images has been used in prior investigations that explored diverse lung parenchyma pathologies, proving that $\mathrm{Al}$ is a suitable tool for supplementing conventional visual assessment ${ }^{15}$. The advantages of quantitative CT Al 
Figure 2. Mortality receiver-operating characteristic curve of computed tomography variables.

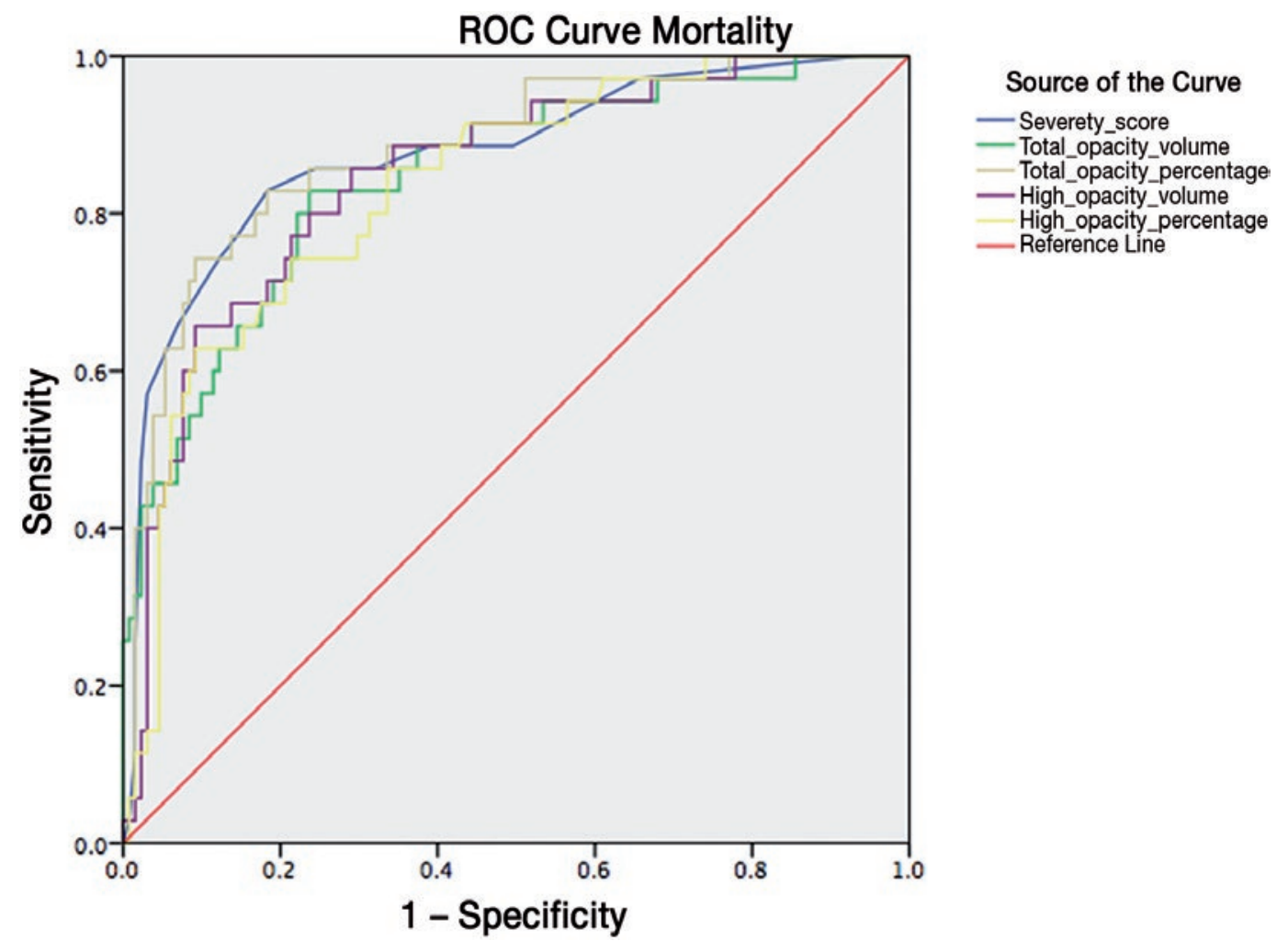

Figure 3. Need for mechanical ventilation receiver-operating characteristic curves of studied computed tomography variables.

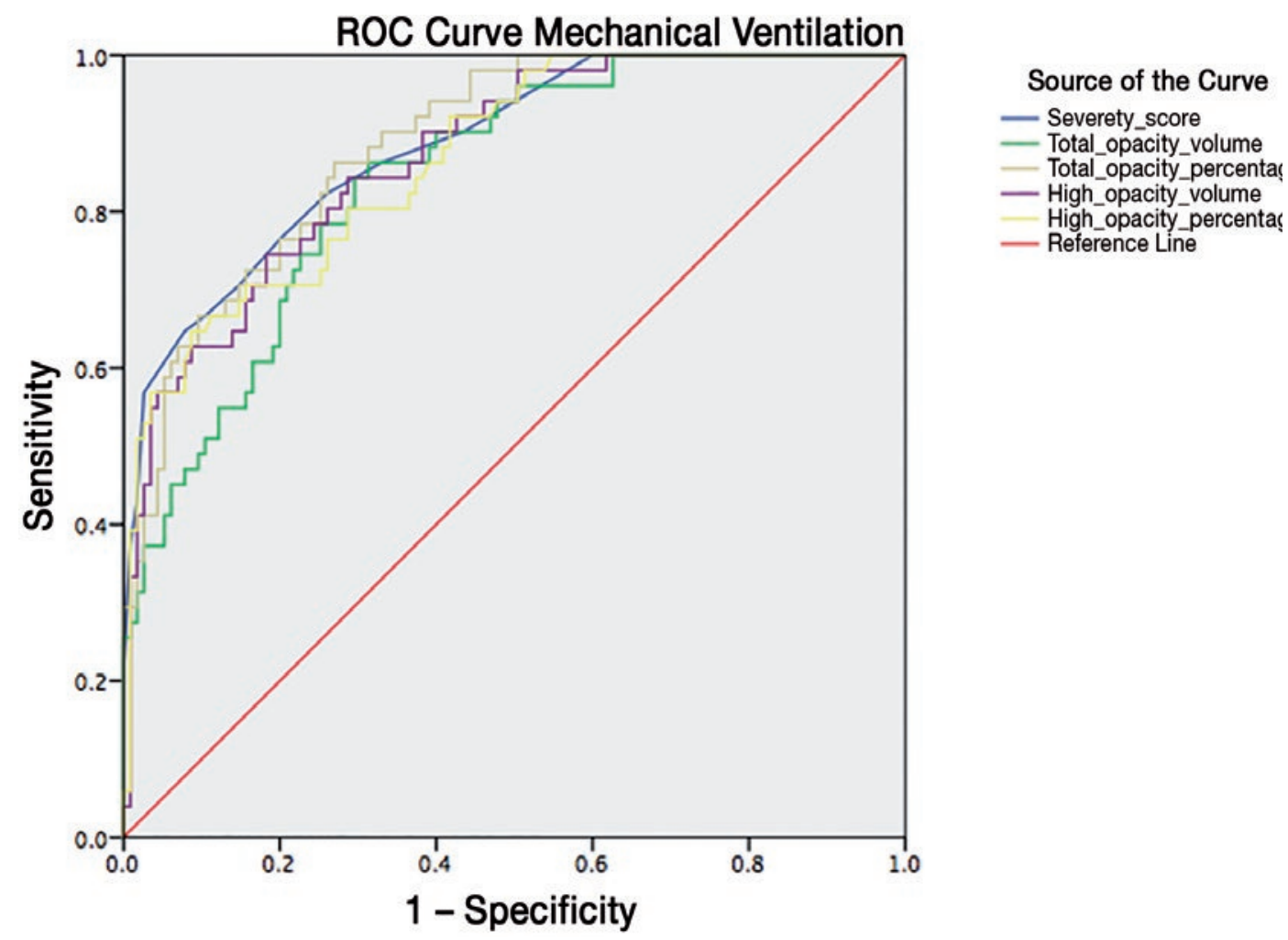


software under radiologist supervision include a significant reduction in interpretation time, fast learning curve, and increased objectivity of the quantitative severity assessment of the affected lung parenchyma which decreases the variability between readers. At present, CT Al software has been tested in research and academic centers under radiologic surveillance with encouraging results. It represents a promising tool for implementation in the clinical field.

This report is one of the first cohort studies to investigate the association between mortality and MV requirement with $\mathrm{Al}$ thoracic CT measures in a Hispanic (Mexican) population. CT-SS and the percentage of total opacity had the strongest diagnostic accuracy estimators, robust enough to maintain a significant association in the logistic models' multivariate adjustment, including the predetermined clinical and laboratory parameters. Thresholds higher than 51\% for the percentage of total opacity and $>10.5$ points in the CT-SS had the highest AUC ( $88 \%$ and $87 \%$, respectively), with the former having a specificity of $91 \%$. Similarly, a threshold of $>25 \%$ had a negative likelihood ratio of 0.18 . This CT Al measure could integrate prognostic tools with laboratory and clinical variables to determine the risks of mortality and MV requirement in hospitalized COVID-19 patients in the present cohort. The predictive values might have been affected by the high mortality in our patients; however, the prevalence was similar to those stated in other reports ${ }^{16}$.

Recently, Lessmann et al. developed an Al system that accurately identified COVID-19 patients with high diagnostic performance and assigned SS in good agreement with the experienced radiologist ${ }^{17}$. Lanza et al. also used computer-aided quantitative analysis of CT images to determine compromised lung volumes and predict the need for oxygenation support and intubation ${ }^{18}$. They found that patients with compromised lung volumes of $>23 \%$ were at risk for intubation. Similarly, in our study, we obtained a threshold of $25 \%$ for patients meeting intubation criteria.

Our logistic regression models for meeting MV criteria and mortality, combining the significant biochemical and tomographic variables, had two significant variables each. Most of the CT variables' adjusted ORs lost significance, as shown by the strong correlation detected. Notably, prognostic (clinical and biochemical) surrogates have shown significant heterogeneity among reports of different COVID-19 populations, showing mixed significance and effects, which is expected in such a new entity ${ }^{19}$.

Nevertheless, our models could distinguish patients' requirements for $\mathrm{MV}$ and risk of mortality with an acceptable $\mathrm{R}^{2}$. The overall performance, discrimination, and calibration statistics were favorable for both models and could be used by clinicians for decisionmaking during hospitalization to predict high-risk patients.

LDH is a widely available, affordable, and rapidly measured biochemical marker that has been associated with severe pneumonia and mortality. Ortiz-Brizuela et al. found that lactate dehydrogenase was significantly higher in patients admitted to the $\mathrm{ICU}^{14}$. Dong et al. found a high accuracy for predicting mortality in critically ill patients ${ }^{20}$ with a $353 \mathrm{U} / \mathrm{L}$ threshold and a hazard ratio of 5.98. Similarly, in a pooled analysis including 1,532 COVID-19 patients, elevated lactate dehydrogenase levels had a 6- and 16-fold increase in odds for severe disease and mortality, respectively ${ }^{21}$. In addition, it has also been evaluated as a potential marker to assess treatment response; increased and decreased levels correlate with radiographic progress or improvement ${ }^{22}$.

Likewise, troponin I is an efficacious, inexpensive, and rapidly measured cardiac injury biomarker. Several studies have identified it as an independent risk factor for the need for mechanical intubation, severe disease, and transfer to the ICU ${ }^{23-25}$. Shah et al. reported OR of 5.18 and 4.95 for MV and admission to the ICU, respectively ${ }^{24}$. In a New York cohort of 2736 hospitalized COVID-19 patients, $36 \%$ had elevated troponin I within $24 \mathrm{~h}$ of admission. Patients with levels higher than $0.09 \mathrm{ng} / \mathrm{mL}$ had a significant mortality risk (adjusted Hazard ratio 3.03$)^{25}$.

In another study that evaluated AI CT software, Zhang et al. proposed a prognostic model for a Chinese population-based on radiographic and biochemical criteria to predict mortality, the clinical need for $\mathrm{MV}$, or the need to be transferred to the ICU, with an AUC of 0.847 for imaging features alone, and 0.909 combined with clinical data ${ }^{26}$. Francone et al. also found that CT Al scores were positively correlated with inflammatory biomarkers and associated higher 
scores with mortality ${ }^{27}$. In conjunction with our findings, these studies support the use of Al to classify risk, monitor patients' response to treatment, and evaluate disease progression in COVID-19, thereby opening a door for $\mathrm{Al}$ to improve resource allocation.

This study has several limitations. This research used a retrospective design. All included patients were from a single tertiary referral center, and only hospitalized patients were enrolled. We analyzed only reconstructions with a slice thickness of $1.5 \mathrm{~mm}$; thinner reconstructions may have a higher diagnostic yield, as the software company recommends. Aside from improving the interobserver agreement mentioned before, Al's performance requires specialized software and has been tested by trained radiologists so far, which might not be widely available.

Further multicenter studies with larger cohorts are encouraged to establish new scenarios for evaluating and externally validating risk prediction performance in similar hospitalized populations. Other quantitative variables obtained by $\mathrm{Al}$ may be studied for their clinical implications as a tool in the patient follow-up, quantifying the disease's possible sequelae. Al can be beneficial in different scenarios throughout the evolution of this pandemic and validation of the information obtained through this tool in our daily practice is imperative.

In conclusion, CT-SS and total opacity percentage had good diagnostic utility for mortality and MV requirement. The proposed prognostic models using variables measured by the $\mathrm{Al}$ software in chest $\mathrm{CT}$ (severity score for meeting MV criteria and percentage of total opacity $>51 \%$ for mortality) and predetermined laboratory elements (troponin I for MV and lactate dehydrogenase for mortality) had good risk classification performance in hospitalized COVID-19 patients, strengthening the evidence for the use of this tool as part of the triage process in the CT analysis of COVID-19 patients.

\section{SUPPLEMENTARY DATA}

Supplementary data are available at Revista de Investigación Clínica online (www.clinicalandtranslationalinvestigation.com). These data are provided by the corresponding author and published online for the benefit of the reader. The contents of supplementary data are the sole responsibility of the authors.

\section{REFERENCES}

1. Chen N, Zhou M, Dong X, Qu J, Gong F, Han Y, et al. Epidemiological and clinical characteristics of 99 cases of 2019 novel coronavirus pneumonia in Wuhan, China: a descriptive study. Lancet. 2020;395:507-13

2. Huang C, Wang Y, Li X, Ren L, Zhao J, Hu Y, et al. Clinical features of patients infected with 2019 novel coronavirus in Wuhan, China. Lancet. 2020;395:497-506.

3. Yang R, Li X, Liu H, Zhen Y, Zhang X, Xiong Q, et al. Chest CT severity score: an imaging tool for assessing severe COVID-19. Radiol Cardiothorac Imaging. 2020;2:e200047.

4. Kucirka LM, Lauer SA, Laeyendecker O, Boon D, Lessler J. Variation in false-negative rate of reverse transcriptase polymerase chain reaction-based SARS-CoV-2 tests by time since exposure. Ann Intern Med. 2020;173:262-7

5. Fang $Y$, Zhang $H$, Xie J, Lin M, Ying L, Pang $P$, et al. Sensitivity of chest CT for COVID-19: comparison to RT-PCR. Radiology. 2020;296:E115-7.

6. Ng MY, Lee EY, Yang J, Yang F, Li X, Wang $H$, et al. Imaging profile of the COVID-19 infection: radiologic findings and literature review. Radiol Cardiothorac Imaging. 2020;2:e200034.

7. Rubin GD, Ryerson CJ, Haramati LB, Sverzellati N, Kanne JP, Raoof S, et al. The role of chest imaging in patient management during the COVID-19 pandemic: a multinational consensus statement from the Fleischner Society. Radiology. 2020; 296:172-80.

8. Revel MP, Parkar AP, Prosch H, Silva M, Sverzellati N, Gleeson F, et al. COVID-19 patients and the radiology departmentadvice from the European Society of Radiology (ESR) and the European Society of Thoracic Imaging (ESTI). Eur Radiol. 2020; 30:4903-9.

9. Bao C, Liu X, Zhang H, Li Y, Liu J. Coronavirus disease 2019 (COVID-19) CT findings: a systematic review and meta-analysis. J Am Coll Radiol. 2020;17:701-9.

10. Ito R, Iwano S, Naganawa S. A review on the use of artificial intelligence for medical imaging of the lungs of patients with coronavirus disease 2019. Diagn Interv Radiol. 2020;26:443-8.

11. Kundu S, Elhalawani H, Gichoya JW, Kahn CE. How might AI and chest imaging help unravel COVID-19's mysteries? Radiol Artif Intell. 2020;2:e200053.

12. Li L, Qin L, Xu Z, Yin Y, Wang X, Kong B, et al. Using Artificial intelligence to detect COVID-19 and community-acquired pneumonia based on pulmonary CT: evaluation of the diagnostic accuracy. Radiology. 2020;296:E65-71.

13. Ohkubo H, Kanemitsu Y, Uemura T, Takakuwa O, Takemura M Maeno K, et al. Normal lung quantification in usual interstitial pneumonia pattern: the impact of threshold-based volumetric $\mathrm{CT}$ analysis for the staging of idiopathic pulmonary fibrosis. PLoS One. 2016;11:e0152505.

14. Ortiz-Brizuela E, Villanueva-Reza M, González-Lara MF, TamezTorres KM, Román-Montes CM, Díaz-Mejía BA, et al. Clinical and epidemiological characteristics of patients diagnosed with $\mathrm{CO}$ VID-19 in a tertiary care center in Mexico city: a prospective cohort study. Rev Invest Clin. 2020;72:165-77.

15. Chen A, Karwoski RA, Gierada DS, Bartholmai BJ, Koo CW. Quantitative CT analysis of diffuse lung disease. Radiographics. 2020;40:28-43.

16. Richardson S, Hirsch JS, Narasimhan M, Crawford JM, McGinn T, Davidson $\mathrm{KW}$, et al. Presenting characteristics, comorbidities, and outcomes among 5700 patients hospitalized with COVID-19 in the New York city area. JAMA. 2020;323:2052-9.

17. Lessmann N, Sánchez Cl, Beenen L, Boulogne LH, Brink M, Calli $\mathrm{E}$, et al. Automated assessment of CO-RADS and chest CT severity scores in patients with suspected COVID-19 using artificial intelligence. Radiology. 2020;202439 [Epub ahead of print].

18. Lanza E, Muglia R, Bolengo I, Santonocito OG, Lisi C, Angelotti $\mathrm{G}$, et al. Quantitative chest CT analysis in COVID-19 to predict the need for oxygenation support and intubation. Eur Radiol. 2020;30:1-9. 
19. Potere $N$, Valeriani E, Candeloro M, Tana M, Porreca E, Abbate $A$, et al. Acute complications and mortality in hospitalized patients with coronavirus disease 2019: a systematic review and meta-analysis. Crit Care. 2020;24:389.

20. Dong X, Sun L, Li Y. Prognostic value of lactate dehydrogenase for in-hospital mortality in severe and critically ill patients with Covid-19. Int J Med Sci. 2020;17:2225-31.

21. Henry BM, Aggarwal G, Wong J, Benoit S, Vikse J, Plebani M, et al. Lactate dehydrogenase levels predict coronavirus disease 2019 (COVID-19) severity and mortality: a pooled analysis. Am J Emerg Med. 2020;38:1722-6.

22. Wu MY, Yao L, Wang Y, Zhu XY, Wang XF, Tang PJ, et al. Clinical evaluation of potential usefulness of serum lactate dehydrogenase (LDH) in 2019 novel coronavirus (COVID-19) pneumonia. Respir Res. 2020;21:171.

23. Liu D, Yang Q, Chen W, Chen H, Feng Y, Hu W, et al. Troponin I, a risk factor indicating more severe pneumonia among patients with novel coronavirus infected pneumonia. Clin Infect Pract. 2020;7:100037.

24. Shah P, Doshi R, Chenna A, Owens R, Cobb A, Ivey $H$, et al Prognostic value of elevated cardiac troponin I in hospitalized Covid-19 patients. Am J Cardiol. 2020;135:150-3.

25. Lala A, Johnson KW, Januzzi JL, Russak AJ, Paranjpe I, Richter F, et al. Prevalence and impact of myocardial injury in patients hospitalized with COVID-19 infection. J Am Coll Cardiol. 2020; 76:533-46

26. Zhang K, Liu X, Shen J, Li Z, Sang $Y, W u$ X, et al. Clinically applicable Al system for accurate diagnosis, quantitative measurements, and prognosis of COVID-19 pneumonia using computed tomography. Cell. 2020;181:1423-33.

27. Francone M, lafrate F, Masci GM, Coco S, Cilia F, Manganaro L, et al. Chest CT score in COVID-19 patients: correlation with disease severity and short-term prognosis. Eur Radiol. 2020; 30:1-10. 